\title{
Organogel formation rationalized by Hansen solubility parameters: influence of gelator structure
}

\author{
Julien Bonnet, ${ }^{\text {a,b }}$ Gad Suissa, ${ }^{\text {a,b }}$ Matthieu Raynal ${ }^{\text {a,b }}$ and Laurent Bouteiller ${ }^{\text {a,b }}$ *
}

\begin{abstract}
${ }_{5}$ Some organic compounds form gels in liquids by forming a network of anisotropic fibres. Based on extensive solubility tests of four gelators of similar structures, and on Hansen solubility parameter formalism, we have probed the quantitative effect of a structural variation of the gelator structure on its gel formation ability. Increasing the length of an alkyl group of the gelator obviously reduces its polarity, which leads to a gradual shift of its solubility sphere towards lower $\delta_{p}$ and $\delta_{h}$ values. At the same time, 10 its gelation sphere is shifted - to a much stronger extent - towards larger $\delta_{p}$ and $\delta_{h}$ values.
\end{abstract}

\section{Introduction}

Organogels are formed by dispersing a small proportion of a low molecular weight gelator (LMWG) in a liquid where it self15 assembles into anisotropic (usually fibrillar) structures, that form an entangled network. ${ }^{1,2}$ These gels are thermally responsive and show a rich variety of rheological properties going from elastic gels to viscoelastic solutions. ${ }^{3-8}$ Such fibrillar structures are highly promising candidates for a wide variety of applications. ${ }^{9-25}$

20 To date, a significant range of LMWGs are known, but most of them have been discovered by serendipity, and their gelation behaviour has been probed through tedious trial and error processes. Among the various attempts to rationalize the gelation ability of LMWGs, we proposed a method based on Hansen ${ }_{25}$ solubility parameters (HSP). ${ }^{26}$ We have shown that it is possible to predict the behaviour of a known LMWG in a new liquid of interest, if the solubility of this particular LMWG has been previously tested in a set of liquids enabling to identify the domain in Hansen space where gels are obtained (the gelation

30 sphere). This approach has been tested by others and appears to be a useful data mining tool, allowing to considerably reduce the number of trials usually involved during the identification of a suitable gelator for a particular application, i.e. in a particular formulation. ${ }^{27-39}$ However, an obvious limitation of this approach 35 is that it requires the prior synthesis and solubility testing of a potential LMWG. It would be much more useful if the concept could be extended to predict the gelation ability of a potential LMWG without having to synthesize it beforehand. While the $a b$ initio prediction of gelation is currently out of reach, a significant 40 step forward would be made if we could predict the gelation ability of a new (untested) compound based on gelation data determined on known and structurally similar compounds. This would enable to efficiently extend the list of LMWGs that are already identified. Therefore, the objective of this study is to ${ }_{45}$ investigate if it is possible to rationalize how the gelation sphere of a particular gelator is affected by small structural variations of the gelator (herein the length of an alkyl side chain). We expect that the collection of such studies will enable to propose rules on how to adapt a known gelator to reach gelation in a liquid of
${ }_{50}$ interest.

\section{Experimental section Synthesis}

The preparation and characterization of amide $\mathbf{C 1 2}$ was reported previously. ${ }^{27}$ Amides C4, C8 and $\mathbf{C 1 8}$ were prepared from $(R)$ 55 12-hydroxystearic acid (12-HSA) and the corresponding amine according to the following procedure. 12-HSA was recrystallized (mp $\left.78.6{ }^{\circ} \mathrm{C}\right){ }^{40}$ To a cooled (at $-5{ }^{\circ} \mathrm{C}$ ) and stirred solution of ethyl chloroformate $(2 \mathrm{~mL}, 21 \mathrm{mmol})$ in dry THF $(50 \mathrm{~mL})$ was added slowly a solution of 12-HSA $(6.43 \mathrm{~g}, 21 \mathrm{mmol})$ and 60 triethylamine $(3 \mathrm{~mL}, 21 \mathrm{mmol})$ in dry THF $(50 \mathrm{~mL})$ while maintaining the temperature at $-5{ }^{\circ} \mathrm{C}$. The mixture was stirred for an additional $40 \mathrm{~min}$. The amine $(24 \mathrm{mmol})$ in $50 \mathrm{~mL}$ dry THF was added to the vigorously stirred solution at $-5{ }^{\circ} \mathrm{C}$, and the reaction mixture was kept at room temperature for $24 \mathrm{~h}$. The ${ }_{65}$ solvent was removed under vacuum and the residue was washed successively with ethyl acetate $(200 \mathrm{~mL}), 3 \mathrm{~N} \mathrm{HCl}(150 \mathrm{~mL})$, aqueous $1 \mathrm{M} \mathrm{Na}_{2} \mathrm{CO}_{3}(200 \mathrm{~mL})$, water $(250 \mathrm{~mL})$ and diethyl ether $(200 \mathrm{~mL})$ and finally dried under vacuum. A white powder was obtained with a yield between 60 and $80 \%$. See Supporting ${ }_{70}$ Information for the NMR $\left({ }^{1} \mathrm{H}\right.$ and $\left.{ }^{13} \mathrm{C}\right)$, FTIR and ESI-TOF characterization.

\section{Gel preparation}

Samples were prepared by introducing $20 \mathrm{mg}$ of gelator and $1 \mathrm{~mL}$ of liquid in a screw-cap vial, heating until dissolution and leaving 75 the vial to cool on the bench. After a few weeks, the aspect of the samples was noted (gel (G), solution (S) or insoluble (I)). This long waiting period was selected because a few samples were observed to evolve slowly even after one day at room temperature.

\section{${ }_{80}$ Results and Discussion}

Various amide derivatives of $(R)$-12-hydroxystearic acid were previously reported to be efficient gelators. ${ }^{40} \mathrm{We}$ therefore synthesized four potential gelators differing only in the length of their alkyl chain (Fig. 1) and tested their gelation ability 
following a previously described two-step procedure. ${ }^{27}$ In the first step, a set of 21 liquids (Table S1) homogeneously scattered in Hansen space were selected to test gel formation of amides $\mathbf{C} 4$ to C18. Based on this preliminary data set, 14 of the previous 5 liquids were selected and mixed together in various proportions to generate 68 liquid mixtures (Table S2) in order to better define the limits of the gelation domain of each LMWG. In summary, the solubility of each LMWG was tested in the same 89 liquids or liquid mixtures. Fig. 2 shows the extended gel formation data 10 obtained after both steps. Qualitatively, it can be seen that liquids with a large hydrogen bonding parameter $\left(\delta_{h}\right)$ and a moderate dipolar interaction parameter $\left(\delta_{p}\right)$ tend to solubilize amide $\mathbf{C 4}$. Increasing the length of the alkyl chain shifts the solubility domain to less polar liquids and also considerably shrinks the size 15 of this solubility domain. This effect is so strong that none of the 89 liquids tested could dissolve amide C18. As far as gelation is concerned, the trend is completely different: amides $\mathbf{C 4}, \mathbf{C 8}$ and C12 gel liquids of low hydrogen bonding parameter $\left(\delta_{h}\right)$, in a large range of dipolar interaction parameter $\left(\delta_{p}\right)$. Actually, the 20 gelation domains of these three compounds look roughly similar, whereas the gelation domain for amide $\mathbf{C 1 8}$ is strongly shifted toward large values of dipolar interaction parameter $\left(\delta_{p}\right)$. We analyse these data in more detail below.

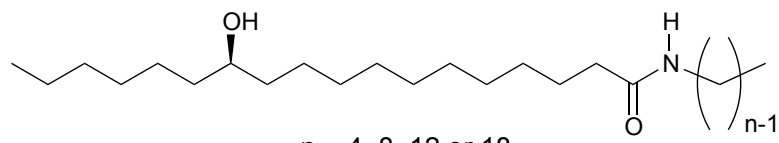

$\mathrm{n}=4,8,12$ or 18

Fig. 1 Structure for LMWGs Cn.

\section{The solubility domain}

The test liquids were initially chosen in order to determine the gelation domain as precisely as possible, without paying attention to whether the non-gelled liquids actually formed solutions or

30 precipitates. Nevertheless, the data can be used, at least qualitatively, to characterize the solubility domain of amides $\mathbf{C 4}$ to C12. Fig. 3 shows on the same plot all the points in Hansen space corresponding to the good solvents of amides $\mathbf{C 4}, \mathbf{C 8}$ and C12. It is obvious that the solubility domain is reduced on going 35 from $\mathbf{C} 4$ to C12. Actually, we can observe that all solvents for $\mathbf{C 1 2}$ dissolve $\mathbf{C 8}$ and $\mathbf{C 4}$ and that all solvents for $\mathbf{C 8}$ dissolve $\mathbf{C 4}$. The number of good solvents for amides C8 and $\mathbf{C 1 2}$ is too low to allow the determination of the solubility sphere with a reasonable precision, ${ }^{26}$ therefore we chose to quantify the 40 solubility domain by calculating the centroid of the solubility domain, computed as the average of the coordinates of the good solvents. Fig. 4 and Table S4 show that the centroid of the solubility domain shifts toward lower values of $\delta_{h}$ and $\delta_{p}$ and that $\delta_{d}$ is little affected when the length of the alkyl chain is ${ }_{45}$ increased. ${ }^{41}$ This result is of course expected since the density of hydrogen bonding and polar groups on the amide is reduced when the length of the alkyl chain is increased. The centroid values are not supposed to be identical to the centre of the solubility sphere because the data points are not perfectly homogeneously spread ${ }_{50}$ over space, which results in a strong sensitivity of the centroid on the particular choice of the test liquids. For the same reason, the centroid value is not supposed to be identical to the solubility parameter of the solute, but it is still informative to compare them. Therefore, the solubility parameters of amides $\mathbf{C 4}$ to $\mathbf{C 1 2}$ ${ }_{55}$ were estimated by a group contribution method $^{42}$ (Table S3) and plotted on Fig. 4. The experimentally determined centroids of the solubility domains are shifted in the same direction as the calculated solubility parameters when the length of the alkyl chain is increased. The centre of the solubility domain thus seems 60 to be directly correlated to the molecular structure of amides $\mathbf{C 4}$ to $\mathbf{C 1 2}$.
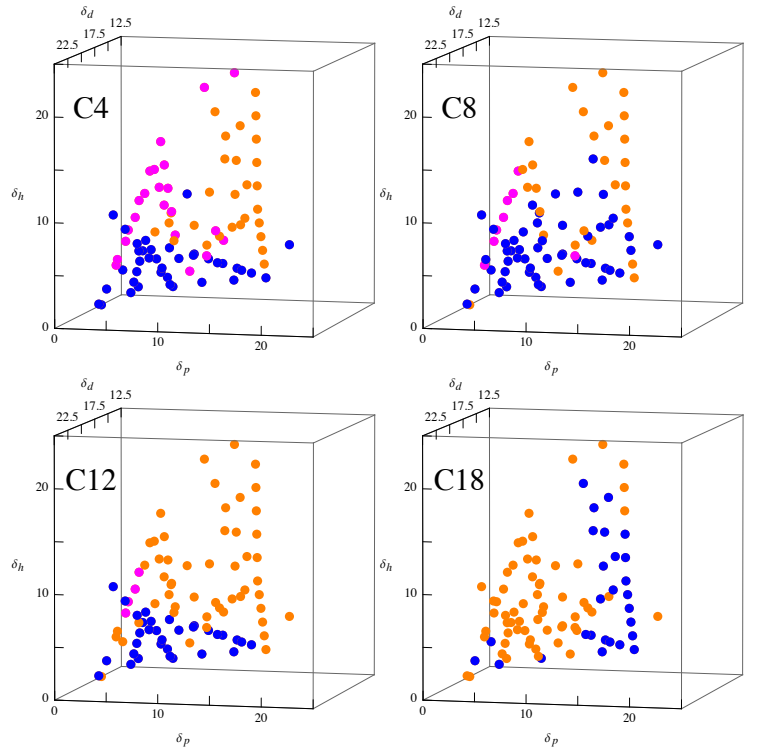

Fig. 2 Solubility data represented in Hansen space for amides $\mathbf{C 4}$ to C18. Blue: gel; magenta: soluble; orange: insoluble.

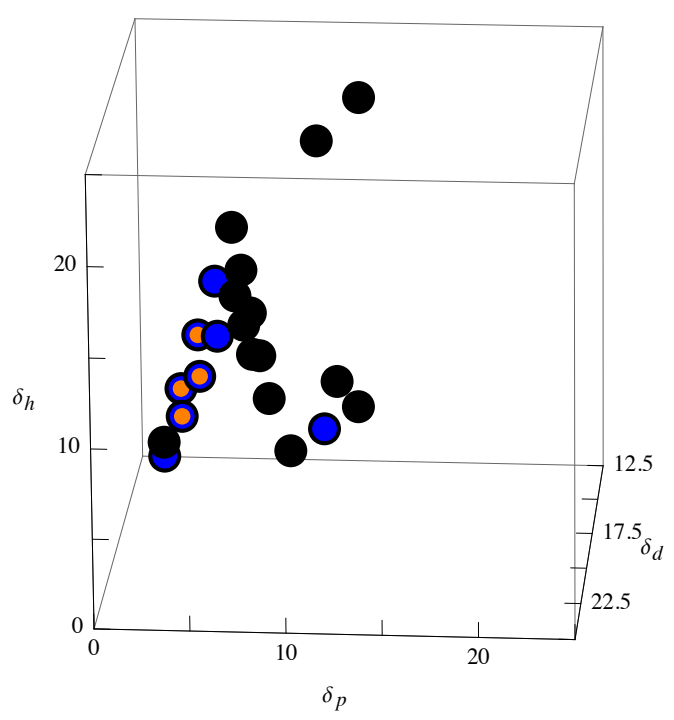

Fig. 3 Solubility data represented in Hansen space. The black (respectively blue or orange) points represent the liquids that dissolve 70 amide $\mathbf{C 4}$ (respectively C8 or $\mathbf{C 1 2}$ ). The graded size of the points allows visualizing liquids that dissolve several amides. 


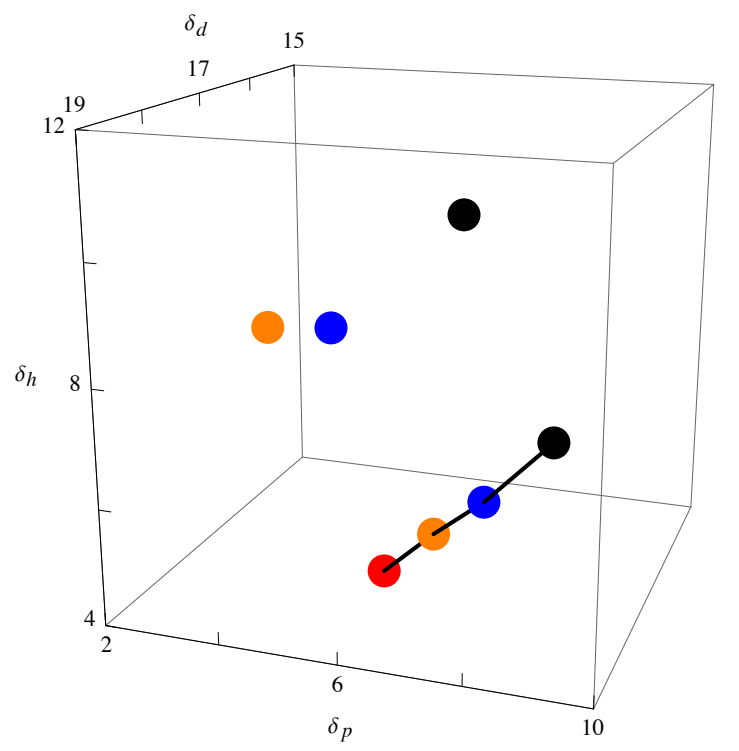

Fig. 4 Solubility data represented in Hansen space. The linked black (respectively blue, orange or red) point represents the calculated

5 solubility parameters for amide C4 (respectively C8, C12 or C18). Each unlinked point represents the centroid for liquids that dissolve each amide.

\section{The gelation domain}

The same approach was then followed to characterize the gelation 10 domains. Fig. 5 shows on the same plot all the points in Hansen space corresponding to the liquids that are gelled by amides $\mathbf{C 4}$ to C18. While most of the liquids that are gelled by amide $\mathbf{C} 4$ have a low hydrogen bonding parameter $\left(\delta_{h}<6 \mathrm{MPa}^{0.5}\right)$, the gelation domain for amide $\mathbf{C 8}$ extends to significantly larger values of $\delta_{h}$.

15 This trend is further amplified in the case of $\mathbf{C 1 8}$ that reaches liquids of very large $\delta_{h}$ and mainly gels liquids of high $\delta_{p}{ }^{43}$ Strangely, the behaviour of amide $\mathbf{C 1 2}$ is not in line with this trend: the gelation domains for amides $\mathbf{C 1 2}$ and $\mathbf{C 4}$ are actually very similar.

20 In order to make these observations more quantitative, the gelation sphere of each LMWG was determined: the idea is to find the centre and the radius of a sphere, so that as many gelled liquids as possible lie inside the sphere, but as many soluble and insoluble samples as possible lie outside. As explained in our ${ }_{25}$ previous publication, ${ }^{27}$ this was performed with the use of HSPiP software. ${ }^{44}$ Figs. S1 to S4 show the obtained gelation spheres: the description of the present data by a sphere is not perfect, but still of practical utility. In the worst case, 13 out of the 89 test liquids are outliers (i.e. gels outside the sphere and non-gels inside the 30 sphere). As shown in Fig. S1, it is possible to significantly improve this description by allowing a second gelation sphere. However, this also adds some complexity in the description of the data. In our hands, the use of a second gelation sphere did not have consequences on the final conclusions from this study, so 35 that we limit the present discussion to the use of a single gelation sphere which describes well more than $85 \%$ of the data points for each LMWG. The centre of the gelation sphere for each amide is plotted on Fig. 6.

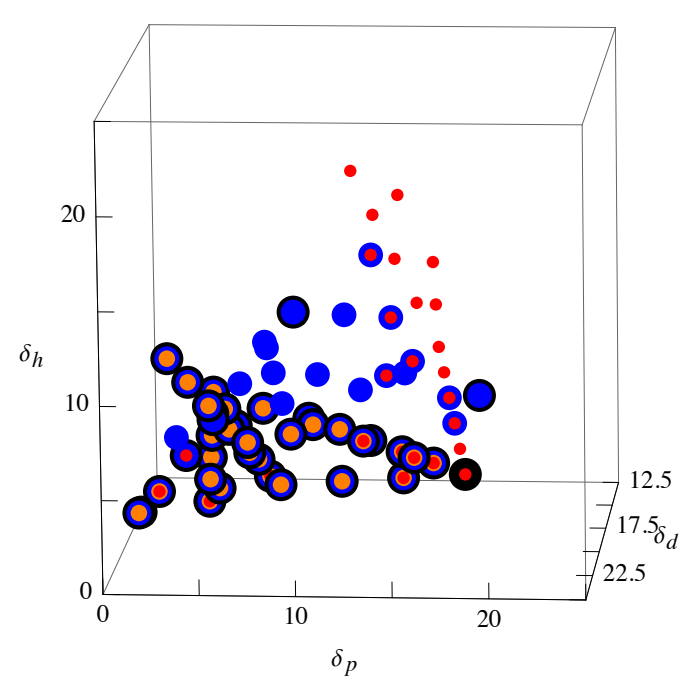

40 Fig. 5 Gelation data represented in Hansen space. The black (respectively blue, orange or red) points represent the liquids that are gelled by amide C4 (respectively $\mathbf{C 8}, \mathbf{C 1 2}$ or $\mathbf{C 1 8}$ ). The graded size of the points allows visualizing liquids that are gelled by several amides.

As in the previous section, the data was also described by 45 calculating the centroid of the gelation domain, computed as the average of the coordinates of the gelled solvents. As explained before, the centroid values are not supposed to be identical to the centre of the gelation sphere (even if all the gelled liquids stand in this gelation sphere). Indeed, the data points are not perfectly ${ }_{50}$ homogeneously spread over Hansen space, so that the centroid strongly depends on the particular choice of the test liquids. Nevertheless, Fig. 6 shows that the centre of the gelation sphere and the centroid of the gelation domain are affected in the same general manner, which means that both approaches can be used to s5 summarize the experimental data. Indeed, both approaches show (Fig. 6 and Tables S5 and S6) that the gelation domain of $\mathbf{C 1 8}$ is strongly shifted toward larger values of all three solubility parameters $\left(\delta_{d}, \delta_{p}\right.$ and $\left.\delta_{h}\right)$ compared to $\mathbf{C 4}$, while $\mathbf{C 8}$ displays intermediate values. This trend is however not followed by $\mathbf{C 1 2}$ ${ }_{60}$ that has $\delta_{p}$ and $\delta_{h}$ values lower than those for $\mathbf{C 8}$. The reason for the different behaviour of amide $\mathbf{C 1 2}$ compared to the others is not clear. However, one possible explanation could be if the molecular packing within the fibres is significantly different for C12 compared to the others. Indeed, several crystalline packing ${ }_{65}$ modes have been reported for this family of LMWGs. ${ }^{40}$

If we now focus on the trend shown by $\mathbf{C 4}, \mathbf{C 8}$ and $\mathbf{C 1 8}$, it is interesting to notice that the gelation domain evolves in the opposite direction from the solubility parameters calculated from a group contribution method (Fig. 6). Indeed, as shown above, the 70 calculated solubility parameters shift toward lower values of $\delta_{h}$ and $\delta_{p}$ when the length of the alkyl chain is increased, whereas gelation domain clearly shifts toward larger values of $\delta_{h}$ and $\delta_{p}$. 
This actually makes sense: it is well known that a polar gelator can only gel low polarity liquids since it will dissolve in polar liquids. Conversely, a non-polar gelator can only gel polar liquids. Therefore, when the number of carbon atoms of the 5 gelator is increased, the gelator becomes less polar and therefore it can gel more polar liquids. More unexpectedly, the magnitude of these shifts are very different: the distance between the gelation domains of $\mathbf{C 4}$ and $\mathbf{C 1 8}$ is quite large (19 $\mathrm{MPa}^{0.5}$ if it is measured through the centre of the gelation spheres or $8 \mathrm{MPa}^{0.5}$ if

10 it is measured through the centroid of the gelation domains) compared to the distance between the calculated solubility parameters of $\mathbf{C 4}$ and $\mathbf{C 1 8}\left(4 \mathrm{MPa}^{0.5}\right)$. This means that the structural variation of the gelator has a proportionate consequence on its solubility, but an amplified consequence on its gelation 15 domain.

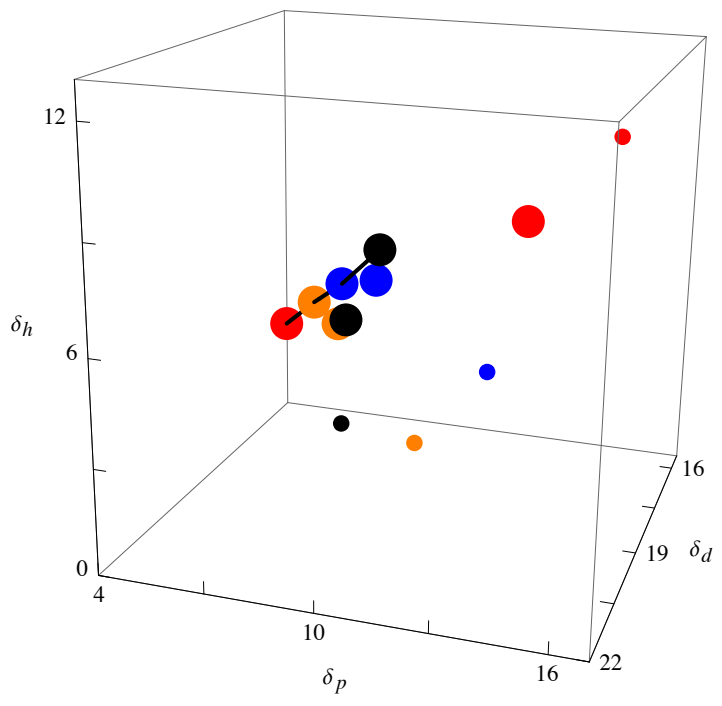

Fig. 6 Gelation data represented in Hansen space. The large linked black (respectively blue, orange or red) point represents the calculated solubility parameters for amide $\mathbf{C 4}$ (respectively $\mathbf{C 8}, \mathbf{C 1 2}$ or $\mathbf{C 1 8}$ ). Each 20 large unlinked point represents the centroid for liquids that are gelled by each amide. Each small point represents the centre of the gelation sphere of each amide.

\section{Conclusion}

Based on extensive solubility tests of four LMWGs having very 25 similar structures (which only differ by the length of their alkyl amide side chain), we have probed the quantitative effect of a structural variation of the gelator structure on its gel formation ability. Increasing the length of an alkyl group of the gelator obviously reduces its polarity, which leads to a gradual shift of its 30 solubility sphere towards lower $\delta_{p}$ and $\delta_{h}$ values. At the same time, its gelation sphere is shifted - to a much stronger extent towards larger $\delta_{p}$ and $\delta_{h}$ values. If further investigations confirm the generality of these observations, they could be the basis for the prediction of the gelation domain for a new LMWG based on 35 data obtained on structurally related compounds.

\section{Acknowledgments}

Financial support from Total company is acknowledged.

\section{Notes and references}

${ }^{a}$ Sorbonne Université, UPMC Univ Paris 06, UMR 8232, IPCM, Chimie 40 des Polymères, F-75005 Paris, France,

${ }^{b}$ CNRS, UMR 8232, IPCM, Chimie des Polymères, F-75005 Paris, France

$\dagger$ Electronic Supplementary Information (ESI) available: Characterization 45 of amides C4 to C18; additional solubility data. See DOI: $10.1039 / \mathrm{b} 000000 \mathrm{x}$

1. Molecular Gels: Materials with Self-Assembled Fibrillar Networks, ed. P. Terech and R. G. Weiss, Springer, Dordrecht, 2006.

50 2. M. George and R. G. Weiss, Acc. Chem. Res., 2006, 39, 489.

3. M. Lescanne, P. Grondin, A. d'Aléo, F. Fages, J.L. Pozzo, O. Mondain Monval, P. Reinheimer and A. Colin, Langmuir, 2004, 20, 3032 .

4. P. Mukhopadhyay, N. Fujita, A. Takada, T. Kishida, M. Shirakawa,

55 and S. Shinkai, Angew. Chem. Int. Ed., 2010, 49, 6338.

5. J. Brinksma, B. L. Feringa, R. M. Kellogg, R. Vreeker and J. van Esch, Langmuir, 2000, 16, 9249.

6. G. Ducouret, C. Chassenieux, S. Martins, F. Lequeux and L. Bouteiller, J. Colloid Interface Sci., 2007, 310, 624.

60 7. B. Isare, L. Petit, E. Bugnet, R. Vincent, L. Lapalu, P. Sautet and L. Bouteiller, Langmuir, 2009, 25, 8400.

8. S. Tang, X. Y. Liu and C. S. Strom, Adv. Funct. Mater., 2009, 19, 2252.

9. L. Bouteiller, Adv. Polym. Sci., 2007, 207, 79.

65 10. A. R. Hirst, B. Escuder, J. F. Miravet and D. K. Smith, Angew. Chem. Int. Ed., 2008, 47, 8002.

11. A. Dawn, T. Shiraki, S. Haraguchi, S.-I. T., and S. Shinkai, Chem Asian J., 2011, 6, 266.

12. J. H. Jung, M. Park and S. Shinkai, Chem. Soc. Rev., 2010, 39, $70 \quad 4286$.

13. M.-O. M. Piepenbrock, G. O. Lloyd, N. Clarke and J. W. Steed, Chem. Rev., 2010, 110, 1960.

14. D. Das, T. Kar and P. K. Das, Soft Matter, 2012, 8, 2348

15. J. Zhang and C.-Y. Su, Coord. Chem. Rev., 2013, 257, 137.

75 16. Z. Zhao, J. W. Y. Lam and B. Z. Tang, Soft Matter, 2013, 9, 4564.

17. S. S. Sagiri, B. Behera, R. R. Rafanan, C. Bhattacharya, K. Pal, I. Banerjee and D. Rousseau, Soft Materials, 2014, 12, 4.

18. D. Dasgupta and J.-M. Guenet, Macromol. Chem. Phys., 2013, 214, 188.

80 19. T. Tu, W. Fang and Z. Sun, Adv. Mat., 2013, 25, 530

20. M. R. Rao and S.-S. Sun, Langmuir, 2013, 29, 15146.

21. M. Yamanaka, J. Incl. Phenom. Macrocycl. Chem., 2013, 77, 3.

22. S. S. Babu, V. K. Praveen and A. Ajayaghosh, Chem. Rev., 2014, 114, 197.

85 23. D. K. Kumar and J. W. Steed, Chem. Soc. Rev., 2014, 43, 2080.

24. K. J. Skilling, F. Citossi, T. D. Bradshaw, M Ashford, B. Kellam and M. Marlow, Soft Matter, 2014, 10, 237.

25. R. G. Weiss, J. Am. Chem. Soc., 2014, 136, 7519.

26. M. Raynal and L. Bouteiller, Chem. Commun., 2011, 47, 8271.

9027 . J. Bonnet, G. Suissa, M. Raynal and L. Bouteiller, Soft Matter, 2014, 10, 3154.

28. P. Curcio, F. Allix, G. Pickaert and B. Jamart-Grégoire, Chem. Eur. J., 2011, 17, 13603.

29. Y. Wu, S. Wu, G. Zou and Q. Zhang, Soft Matter, 2011, 7, 9177.

95 30. J. Gao, S. Wu and M. A. Rogers, J. Mater. Chem., 2012, 22, 12651.

31. H. Xu, J. Song, T. Tian and R. Feng, Soft Matter, 2012, 8, 3478.

32. V. C. Edelsztein, A. S. Mac Cormack, M. Ciarlantini and P. H. Di Chenna, Beilstein J. Org. Chem., 2013, 9, 1826.

33. N. Yan, Z. Xu, K. K. Diehn, S. R. Raghavan, Y. Fang and R. G. Weiss, Langmuir, 2013, 29, 793.

34. N. Yan, Z. Xu, K. K. Diehn, S. R. Raghavan, Y. Fang, and R. G. Weiss, J. Am. Chem. Soc., 2013, 135, 8989.

35. K. K. Diehn, H. Oh, R. Hashemipour, R. G. Weiss and S. R. Raghavan, Soft Matter, 2014, 10, 2632. 
36. Y. Lan, M. G. Corradini and M. A. Rogers, Cryst. Growth Des., 2014, 14, 4811.

37. Y. Lan, M. G. Corradini, X. Liu, T. E. May, F. Borondics, R. G. Weiss and M. A. Rogers, Langmuir, doi: 10.1021/la5008389.

538. Y. Yin, Z. Gao, Y. Bao, B. Hou, H. Hao, D. Liu and Y. Wang, Ind. Eng. Chem. Res., 2014, 53, 1286.

39. H. Shen, L. Niu, K. Fan, J. Li, X. Guan and J. Song, Langmuir, 2014, 30, 9176.

40. V. A. Mallia, M. George, D. L. Blair and R. G. Weiss, Langmuir, $10 \quad 2009,25,8615$.

41. Comparing the centroid of the solubility (or gelation) domains of several compounds is possible because the set of test liquids was the same for all compounds. If this were not the case, a bias would be introduced by the choice of the test liquids.

15 42. J. Brandrup, E. H. Immergut and E. A. Grulke, Polymer Handbook, Wiley, New York, fourth edn, 1999.

43. In fact, a few liquids of low $\delta_{h}$ and $\delta_{p}$ (toluene, chlorobenzene, chloropentane) are also gelled by $\mathbf{C 1 8}$. These liquids are gelled by all four gelators.

20 44. S. J. Abbott, C. M. Hansen, H. Yamamoto, Hansen Solubility Parameters in Practice software, eBook, datasets, www.hansensolubility.com 